\title{
Inclusion Complex of Fexofenadine Hydrochloride with Cyclodextrins
}

\author{
Melita Huremovic ${ }^{1,}$ *, Majda Srabovic ${ }^{1}$, Mirsada Salihovic ${ }^{2}$ and Ekrem Pehlic ${ }^{3}$ \\ ${ }^{1}$ University of Tuzla, Faculty of Science, Organic Chemistry, Tuzla Bosnia and Herzegovina \\ ${ }^{2}$ University of Sarajevo, Faculty of Farmacy Sarajevo, Bosnia and Herzegovina \\ ${ }^{3}$ University of Bihac, Faculty of Health Studies, Bihac, Bosnia and Herzegovina
}

\begin{abstract}
Fexofenadine hydrochloride (FFN), ( \pm )-4-[1-hydroxy-4[4-(hydroxydiphenylmethyl)-1-piperidinyl]butyl] $\alpha, \alpha$-dimethylbenzeneacetic acid hydrochloride, is a second-generation antihistamine that is used to treat allergies. The drug is highly hydrophobic and slightly soluble in water. Cyclodextrins are widely used to improve the physicochemical and pharmaceutical properties such as solubility, stability, and bioavailability of poorly soluble drug molecules. Cyclodextrins can molecularly encapsulate various drugs into their hydrophobic cavity without forming any covalent bonds. Cyclodextrin (CDs), especially $\beta$-Cyclodextrin ( $B-C D)$, are widely used in the pharmaceutical field due to its ability to stabilize drug molecules and taste masking purposes.
\end{abstract}

The phase solubility study was performed according to the method of Higuchi and Connors by adding the fexofenadine hydrochloride in excess to different concentrations of cyclodextrin solutions. Phase solubility study records show that the stability constant and complex stoichiometry of FFN-CD complexes increases linearly with $\mathrm{CD}$ concentration. Also, an increase in the concentration of $\beta$-cyclodextrin leads to an increase in the aqueous solubility of FFN. Complexes were analyzed by UV-VIS spectroscopy using the calibration curve of FFN. Also, UV-VIS spectra indicate a bathochromic shift which proves that complex formation has occurred.

Solid inclusion complexes of fexofenadine/ $\beta$-cyclodextrin and its derivatives were prepared at the molar ratios of 1:1 by the physical mixing method. Characterization of the complexes was performed by using infrared spectroscopy.

Keywords: Fexofenadine hydrochloride; Solubility; Inclusion complex; Cyclodextrins.

\section{Introduction}

Fexofenadine hydrochloride is a pharmaceutically active compound which, as a derivative of piperidine, belongs to the group $\mathrm{H} 1$ of antihistamine pharmaceutically active compounds and its IUPAC name is: ( \pm )-4-[1-hydroxy-4[4-(hydroxydiphenylmethyl)-1-piperidinyl]-butyl] $\alpha, \alpha$-dimethylbenzene-

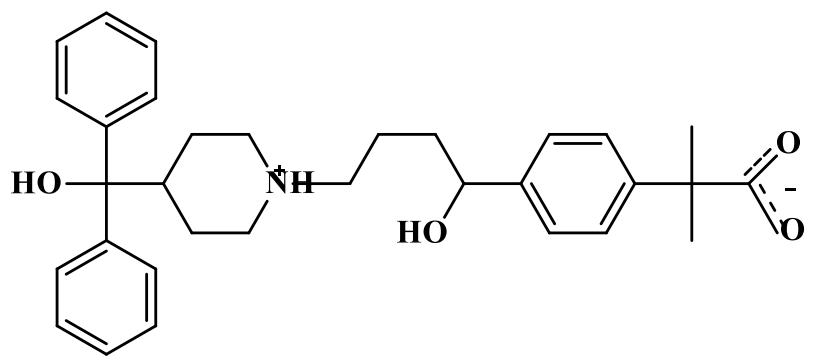

acetic acid hydrochloride. Piperidine belongs to the six-membered heterocyclic amines and is often used as a reagent in organic syntheses, including synthesizing pharmaceutically active ingredients ${ }^{1}$. One of the disadvantages of the oral route of drug administration is low bioavailability, as most drugs have poor solubility in water.

Figure 1. ( \pm )-4-[1-hydroxy-4[4-(hydroxydiphenylmethyl)-1-piperidinyl]-butyl] $\alpha, \alpha$-dimethylbenzeneacetic acid hydrochloride 
In the case of poorly water-soluble drugs, dissolution is the rate-limiting step in the process of drug absorption. Recently, considerable attention has been paid to the importance of testing the solubility of pharmaceutically active ingredients. In vitro solubility tests are used to increase pharmaceutical products' quality and contribute to new formulations ${ }^{2}$. Fexofenadine hydrochloride has several functional groups with the ability to form hydrogen bonds and, under normal conditions, exists as a zwitterion and has the following structure ${ }^{3}$.

Fexofenadine hydrochloride is the synthetic hydrochloride salt of fexofenadine, the carboxylic acid metabolite of terfenadine. High plasma concentrations of terfenadine have been associated with rare incidences of cardiac arrhythmias, and this was the reason for the withdrawal of the drug from clinical use. Fexofenadine hydrochloride has been promoted as terfenadine's replacement. It is an orally active non-sedating $\mathrm{H}_{1}$-receptor antagonist and effectively relieves symptoms associated with seasonal allergic rhinitis. It is superior to most other antihistamines as it does not cross the blood-brain

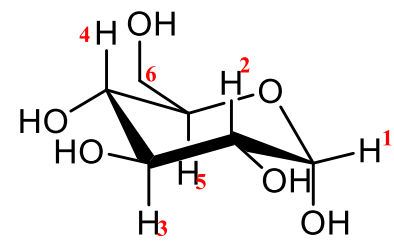

barrier and is safe. But Fexofenadine hydrochloride is very slightly soluble in water, and poor aqueous solubility of a drug is the factor that limits its development into the desired formulation. The absorption of such drugs may be incomplete and variable, and hence there will be variations in the effectiveness ${ }^{4}$. The present work was designed to improve the aqueous solubility of Fexofenadine Hydrochloride.

Cyclodextrins are a group of structurally related natural products formed during the bacterial digestion of cellulose. These bucket-shaped cyclic oligosaccharides comprise a (1-4)-linked $\alpha$-Dglucopyranose units and have a lipophilic central cavity and a hydrophilic outer surface (Figure 3). The most common naturally occurring cyclodextrins are $\alpha$-cyclodextrin, $\beta$-cyclodextrin, and $\gamma$-cyclodextrin, comprising six, seven, and eight glucopyranose units, respectively. Table 1 Given the chair conformation of the glucopyranose units, cyclodextrins are shaped like a truncated cone rather than a perfect cylinder (Figure 2).

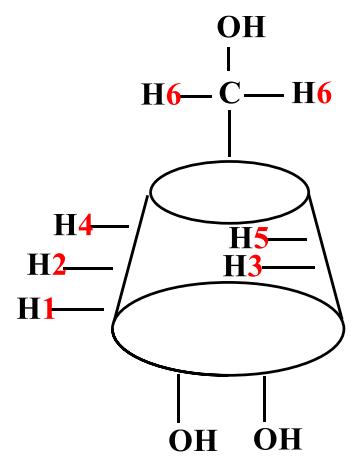

Figure 2. Monomer unit of cyclodextrin ( $\alpha$-D-glucose) and positions of hydroxyl groups in polymer (cyclodextrin)

The hydroxyl functions are oriented to the cone exterior with the primary hydroxyl groups of the sugar residues at the narrow edge of the cone and the secondary hydroxyl groups at the broader edge (Figure 2). The non-polarity of the inner cavity of the cyclodextrin is ideal for the solubilization of nonpolar substances. At the same time, the polarity of the outer part allows its solubility in water and the solubility of the "guest" molecule ${ }^{5}$. The inside of the cyclodextrin molecule is fixed in size and rigid in shape. Thus, a cyclodextrin molecule can only accept a substance whose size corresponds to the frame of the cavity. Cyclodextrins with seven glucose molecules or $\beta$-cyclodextrins are the most commonly used.

Table 1. Structural characteristics of cyclodextrins.

\begin{tabular}{|c|c|c|c|}
\hline Cyclodextrins & $\boldsymbol{\alpha}$ & $\boldsymbol{\beta}$ & $\boldsymbol{\gamma}$ \\
\hline Number of glucose units & 6 & 7 & 1297 \\
\hline Molecular weight & 972 & 1135 & 275 \\
\hline Melting point $\left({ }^{\circ} \mathrm{C}\right)$ & 275 & 280 & 17 \\
\hline Outer diameter $(\AA)$ & 14 & 15 & 10 \\
\hline Large inside diameter $(\AA)$ & 6 & 8 & 9 \\
\hline Small inside diameter $(\AA)$ & 5 & 7 & 8 \\
\hline Cavity Height $($ depth) $(\AA)$ & 8 & 8 & 510 \\
\hline Cavity Volume $(\AA)$ & 176 & 346 & \\
\hline
\end{tabular}




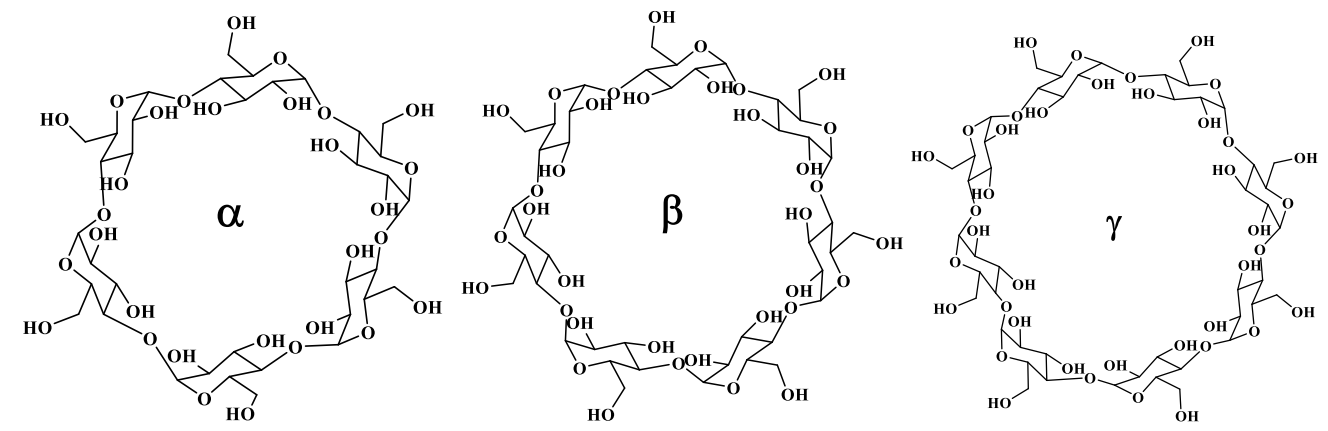

Figure 3. Cyclodextrin structures

Natural cyclodextrins can be modified by substituting some or all of the hydroxyl groups of glucose molecules.

It is generally assumed that when a drug forms a complex with cyclodextrin, the molecule, in part or in whole, is taken up into the somewhat lipophilic cyclodextrin central cavity. In other words, an inclusion complex of the drug and cyclodextrin is formed ${ }^{6}$. Many such cyclodextrin derivatives show significantly better solubility than their natural precursors. As already mentioned, the interior of the cyclodextrin molecule is non-polar, and its size is fixed. Therefore, there are two conditions for the solute to form a complex with cyclodextrin:

- The solute, or at least most of it, must correspond to its size inside the cyclodextrin cavity

- The solute, or at least most of it, must be sufficiently non-polar to displace water into the cyclodextrin cavity

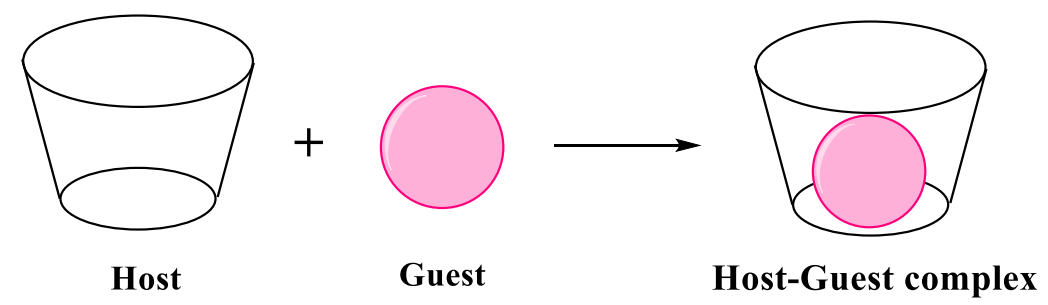

Figure 4. Molecular interaction host-guest

In aqueous solutions, cyclodextrins can form inclusion complexes with pharmaceutically active compounds by taking a molecule of the substance or a lipophilic part thereof into its central cavity. As a result, there is no formation or breaking of covalent bonds, and the substance molecules establish a rapid equilibrium with the free molecules in the solution.
The physicochemical characteristics of the free biologically active substance differ from those associated with cyclodextrin molecules. The same goes for cyclodextrin molecules. If one molecule of the drug forms a complex with one molecule of cyclodextrin, then it is:

$$
\boldsymbol{K} \boldsymbol{c}=\frac{[\mathrm{D}-\mathrm{CD}]}{[\mathrm{D}][\mathrm{CD}]}
$$

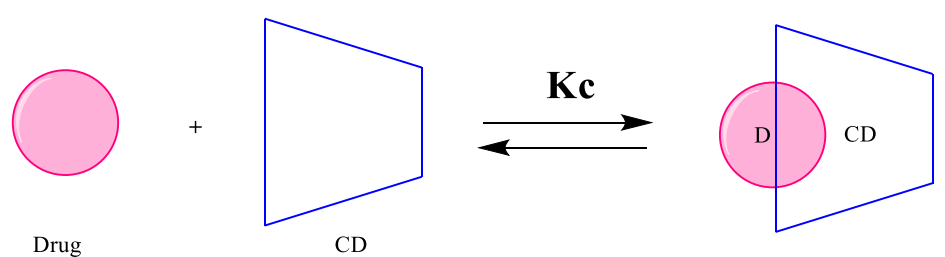

Figure 5. Binding constant of the inclusion complex

Higuchi and Connors ${ }^{7}$ classified complexes based on solubility into multiple phase solubility profiles shown in Figure 6.

According to Higuchi and Connors, phase-solubility diagrams show how the total drug solubility changes with increasing $\mathrm{CD}$ concentration.

A-type diagrams are formed when the drug/CD complex is soluble in the aqueous complexation media, and they are usually associated with the water-soluble $\mathrm{CD}$ derivatives. B-type diagrams are observed when the complex has limited solubility in the media. These are generally associated with the natural CDs with limited solubility in aqueous media, as shown in Figure 6. $\mathrm{A}_{\mathrm{L}}$ represents linear diagram; $A_{P}$ is positive from linearity; $A N$ is negative. $\mathrm{B}_{\mathrm{S}}$ : the complex has some but limited solubility; $\mathrm{B}_{\mathrm{I}}$ : the complex is insoluble. All three 
curves (Figure 6) indicate that water-soluble complexes are being formed with higher solubilities than the uncomplexed substrate. If the slope is lower than unity, a 1:1 complex is formed. If the slope is greater than unity, higher-order complexes are assumed to be involved in the solubilization process.
Although a slope of less than unity does not exclude the possibility of higher-order complexes, a 1:1 complex is often assumed to form in the absence of other information ${ }^{5}$.

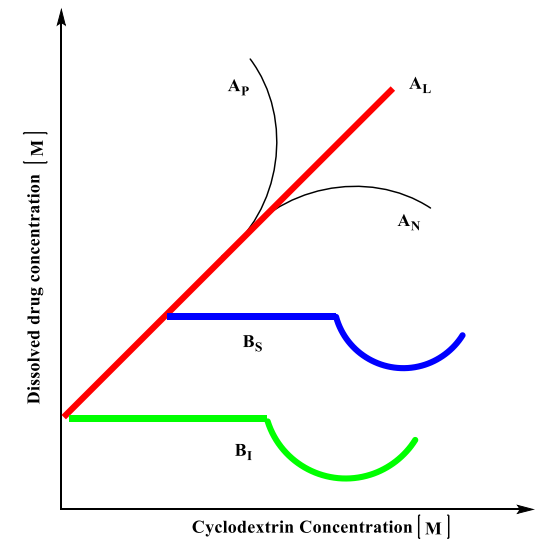

Figure 6. Phase solubility profiles and classification of complexes according to Higuchi and Connors

Many biologically active substance-cyclodextrin complexes were considered to be inclusion complexes. Still, cyclodextrins are known to form both non-inclusive complexes and complex aggregates that can dissolve the substance using micelle-like structures. Phase solubility profiles do not confirm the formation of the inclusion complex. Instead, they describe only how an increase in the cyclodextrin concentration affects the solubility of the biologically active substance ${ }^{8}$.

The constant stability $\mathrm{K}_{1: 1}$ can be calculated from the slope and intrinsic solubility of the biologically active substance $S_{0}$ in an aqueous complexation medium. Intrinsic solubility is the solubility of the substances when cyclodextrin is not present, and it is the point at which the phase solubility curve intersects the y-axis.

\section{$K_{1: 1}=$ slope / [So (1-slope) $]$}

The constant stability values of the $\mathrm{K}_{1: 1}$ complex usually range between 50 and $2000 \mathrm{M}^{-1}$

\section{Experimental}

\subsection{Chemicals}

In this study, the following chemicals were used for experimental work:

- Fexofenadine Hydrochloride, provided by "Bosnalijek" Sarajevo

- $\beta$-cyclodextrin, Sigma Aldrich

- Hydroxy- $\beta$-cyclodextrin, Sigma Aldrich

- Methyl- $\beta$-cyclodextrin, Sigma Aldrich

- Methanol, Merck

- Ethanol, Merck

All used chemicals are of analytical grade.

\subsection{Apparatus}

- Phase solubility measurements were done on a UV-spectrophotometer Perkin Elmer Lambda 25 based on a previously constructed calibration curve. UV/VIS spectroscopy is used for analytical purposes to quantify various analytes, such as transition metalmetal ions, conjugated organic compounds, organic substances, and biochemical macromolecules. Determination usually takes place in solutions. For example, organic substances, especially those with a high degree of conjugation, absorb light in the UV or visible region of the electromagnetic spectrum.

- Qualitative interpretation of the complex was performed with FTIR-spectrophotometer Perkin Elmer 1000; Fourier transforms spectrophotometers significantly improved the quality of the infrared spectrum, accelerated the analysis process, and enabled comparison with the database. In this way, they allowed greater sensitivity and minimal sample quantities. Infrared spectra of the samples were recorded at a resolution of $4 \mathrm{~cm}-1$ and an interval of $4000-400 \mathrm{~cm}^{-1}$. The sample is prepared by taking $10 \mathrm{mg}$ of the sample, which is well powdered and homogenized, with about $90 \mathrm{mg}$ of pure and well dried $\mathrm{KBr}$. $\mathrm{KBr}$ must be dry during the preparation of the sample because otherwise, bands originating from water appear on the spectrum. The resulting mixture is filled into a metal cuvette placed in an FTIR spectrometer, and the powder component's IR spectrum is recorded.

\subsection{Experimental procedure}

\subsubsection{Phase solubility studies}

Samples were prepared according to the method of Higuchi and Connors by adding the pharmaceutically active substance in excess to different concentrations of cyclodextrin solutions ${ }^{7}$. The solubility assay was performed in triplicate. A series of solutions of 
different cyclodextrins of concentration ranging from 0,05-16 mM was added in water. Each of these solutions $(10 \mathrm{ml})$ was added into a series of $50 \mathrm{ml}$ stoppered conical flasks. A constant amount of pure fexofenadine hydrochloride (10mg) was accurately weighed and suspended into each solution.

The samples were left to stir continuously for
72 hours at room temperature $\left(25^{\circ} \mathrm{C}\right)$ and filtered through $0.45 \mu \mathrm{m}$ filter paper. Fresh samples were used to measure the phase solubility on a UVspectrophotometer with a preliminary dilution corresponding to the interval of the calibration curve based on which the concentration is measured (Figure 7).

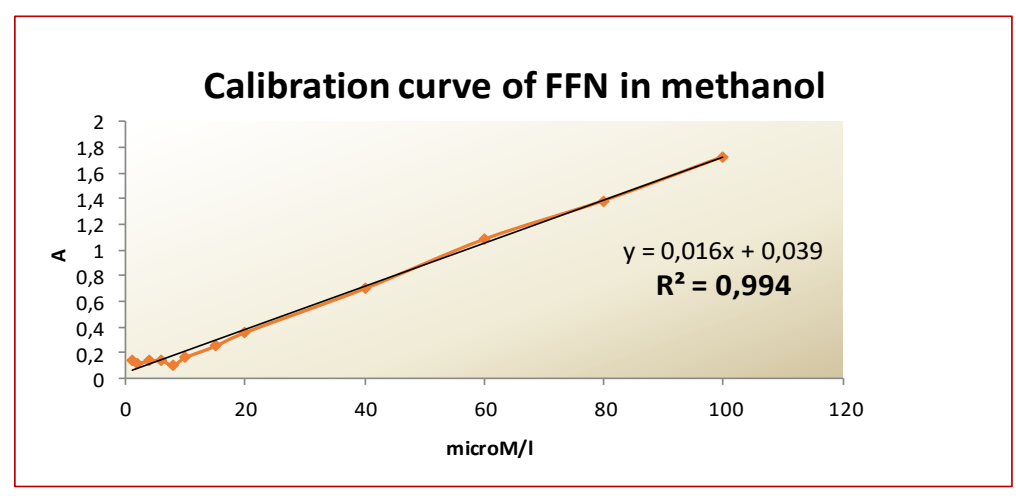

Figure 7. Calibration curve of Fexofenadine Hydrochloride in methanol

\begin{abstract}
2.3.2. Preparation of physical mixtures fexofenadine hydrochloride/cyclodextrin

Fexofenadine Hydrochloride and $\beta-\mathrm{CD}$ were physically mixed in the mortar for $30 \mathrm{~min}$ in the molar ratios of 1:1 and was sealed in a glass ampule and heated at $50^{\circ} \mathrm{C}$. The powder of the physical mixture was left in a desiccator containing anhydrous silica gel. After drying, a FTIR analysis of samples was performed.
\end{abstract}

\section{Results and Discussion}

Table 2. Basic characteristics of fexofenadine hydrochloride.

\begin{tabular}{|c|c|}
\hline Molecular formula & $\mathrm{C}_{32} \mathrm{H}_{39} \mathrm{NO}_{4} \cdot \mathrm{HCl}$ \\
\hline Molecular weight & $538,13 \mathrm{~g} / \mathrm{mol}$ \\
\hline Aqueous solubility & $2,136 \mathrm{mg} / \mathrm{cm}^{3}$ \\
\hline Color & white powder \\
\hline
\end{tabular}

Table 3. USP Solubility criteria ${ }^{9}$.

The aqueous solubility of Fexofenadine Hydrochloride is very poor. Table 2 shows the essential physicochemical characteristics of fexofenadine hydrochloride. Therefore, it is of great importance to increase the aqueous solubility of the drug for better efficacy and chemical availability. Therefore, USP solubility criteria are given in Table 3 .

The use of $\beta-C D$ as a natural cyclodextrin and its synthetic derivatives, 2 -hydroxy- $\beta$-cyclodextrin and methyl- $\beta$-cyclodextrin, increases the aqueous solubility of fexofenadine hydrochloride.

\begin{tabular}{|c|c|}
\hline \multicolumn{2}{|c|}{ USP Solubility criteria } \\
\hline Descriptive Term & $\mathrm{mg} / \mathrm{ml}$ \\
\hline Very soluble & $\geq 1000$ \\
\hline Freely soluble & $1000-100$ \\
\hline Soluble & $100-33$ \\
\hline Sparingly soluble & $33-10$ \\
\hline Slightly soluble & $\mathbf{1 0 - 1}$ \\
\hline Very slightly soluble & $1-0,1$ \\
\hline Practically insoluble & $\leq 0,1$ \\
\hline
\end{tabular}


Figure 8 shows the effect of different concentrations of $\beta$-cyclodextrin on the phase solubility of fexofenadine hydrochloride in an aqueous medium. Diagram in Figure 8a shows $A_{L}$ type phase solubility and the possibility of forming a 1:1 stoichiometric complex because it is slope lower than unity. The constant stability $\mathrm{K}_{1: 1}$ is calculated from Higuchi and Connors equation using parameters obtained from the phase solubility diagram.

The stability constant for samples of fexofenadine hydrochloride- $\beta$-cyclodextrin prepared in aqueous solution is $\mathrm{K}_{1: 1}=114.96 \mathrm{M}^{-1}$. The intrinsic solubility of FFN is presented in the diagram (Figure 8a), and

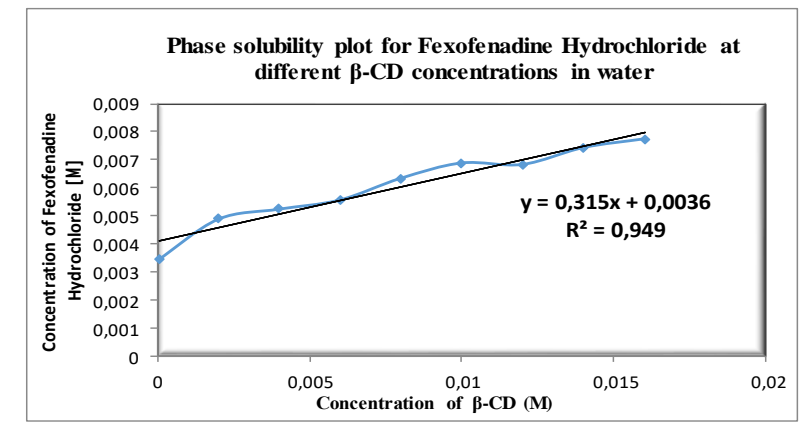

(a) it has a value that corresponds to the aqueous solubility of FFN. Figure 8b show UV-VIS spectra of FFN- $\beta-C D$ complexes. The sample was scanned between $200 \mathrm{~nm}$ to $300 \mathrm{~nm}$ in spectrum mode. FFN has an absorbance maximum at $205 \mathrm{~nm}$, and the spectrum shows bathochromic and hyperchromic shifts due to the presence of auxochromic groups such as cyclodextrin-derived hydroxyl groups. This is a confirmation that a complex has formed between FFN and $\beta-C D$. Since the inner cavity of the cyclodextrin is of a larger diameter, it is possible to form complexes on different parts of the fexofenadine hydrochloride molecule.

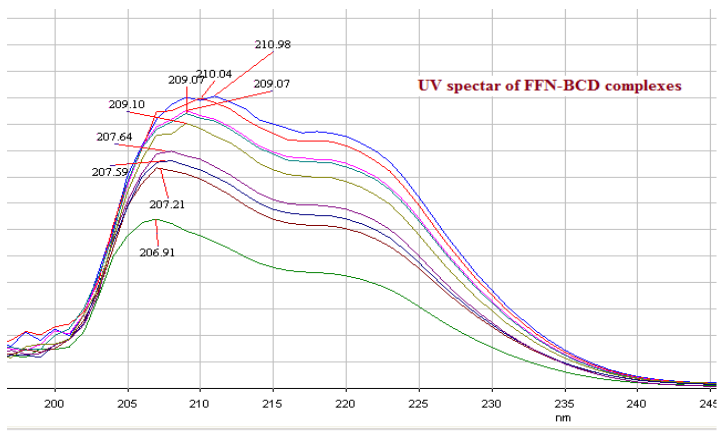

(b)

Figure 8. (a) Phase solubility plot for Fexofenadine Hydrochloride at a different $\beta$-cyclodextrin concentration in water; (b) UV-VIS spectra of Fexofenadine Hydrochloride at a different $\beta$-cyclodextrin concentration in water

Since these are hydrophobic interactions formed between the host and guest molecules, parts of the fexofenadine molecule containing aromatic benzene are included so that three ways of complex formation are possible, two forms involving azacyclonole benzene nuclei and a third involving a benzene nucleus that is located on the aliphatic part of the chain of the molecule 10,11 .<smiles>CC(C)(C)c1ccc(C(C)(C)C(C)(C)C(C)(C)C(=O)O)cc1</smiles>

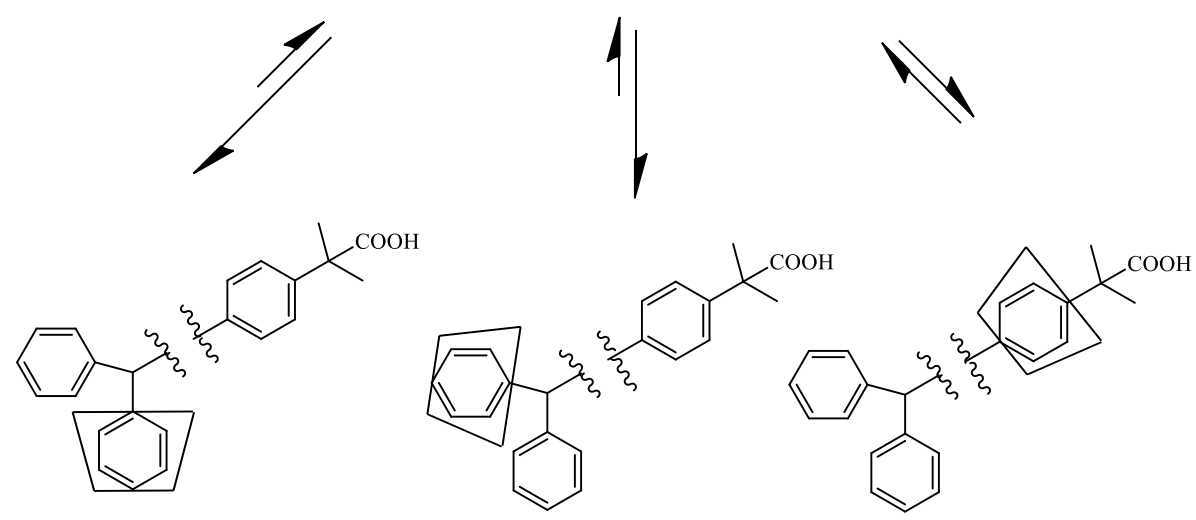

Figure 9. Possible ways of formation of 1:1 complex of fexofenadine hydrochloride- $\beta$-cyclodextrin

2-hydroxypropyl- $\beta$-cyclodextrin is a non-toxic solubilizer. The solubility of the substance increases linearly with the increasing concentration of cyclodextrin. The formation of the substance/cyclodextrin complex takes place very quickly and is a reversible reaction so that the 
complex exists in both aqueous and crystalline forms.

Solutions of many such complexes can be lyophilized to form easily soluble powders that can be compressed into tablets.

Figure 10.a shows the phase solubility profile of fexofenadine hydrochloride with 2-hydroxypropyl- $\beta$ cyclodextrin. Figure 9 shows that this is an $A_{P}$ type of phase solubility according to Higuchi and
Connors, with a possibility of forming a 1:1 complex and a 1:2 complex with cyclodextrin. Also, as a confirmation, there is a high value of the stability constant (K1:1=7325,76 M-1) for fexofenadine samples of fexofenadine hydrochloride-2-Hydroxypropyl- $\beta$-cyclodextrin prepared in aqueous solution. This indicated that UV-VIS spectra show the shift towards higher wavelengths as it is presented in (Figure 10.b).

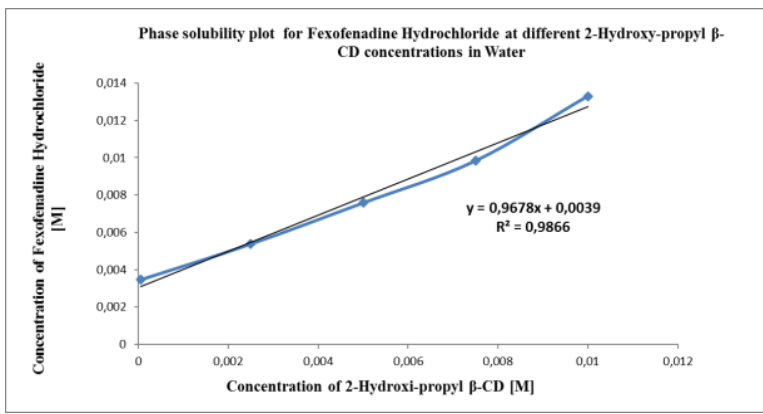

(a)

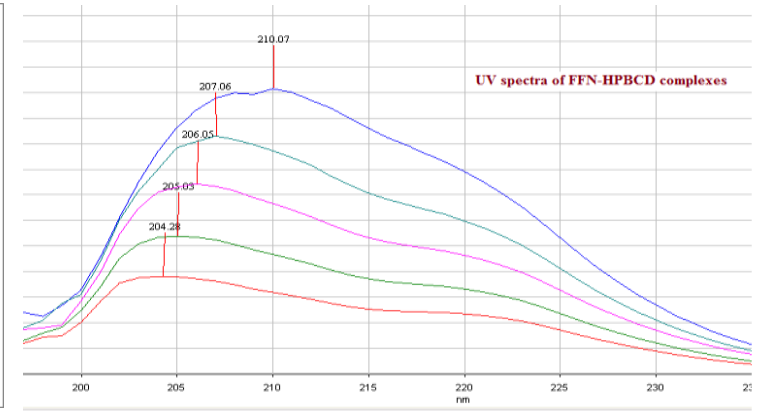

(b)

Figure 10. (a) Phase solubility plot for Fexofenadine Hydrochloride at different 2-Hydroxypropyl- $\beta$ cyclodextrin concentrations in water; (b) UV-VIS spectra of Fexofenadine Hydrochloride at a different 2Hydroxypropyl- $\beta$-cyclodextrin concentration in water

According to Higuchi and Connors, the phase solubility of fexofenadine hydrochloride with methyl- $\beta$-cyclodextrin shows Ap type curve. (Figure 11) Phase solubility diagram, UV-VIS spectra, and value stability constant $(\mathrm{K} 1: 1=2301$
M-1) establish the FFN- methyl- $\beta$-cyclodextrin complex formation. UV-VIS spectra increase absorption maximum with increasing CDconcentration and a bathochromic shift.

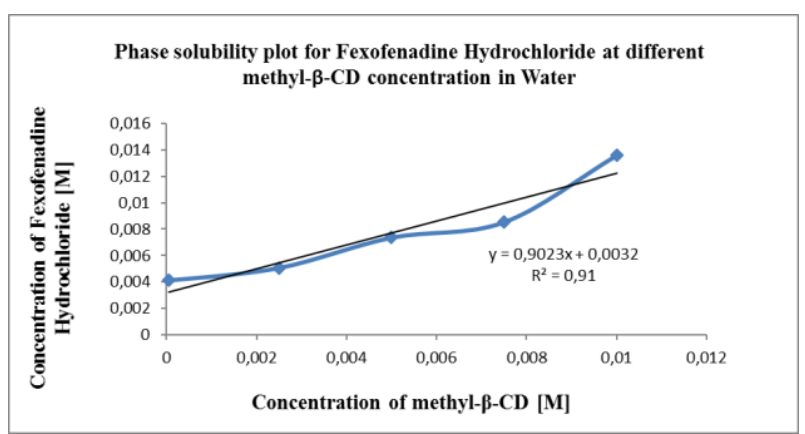

(a)

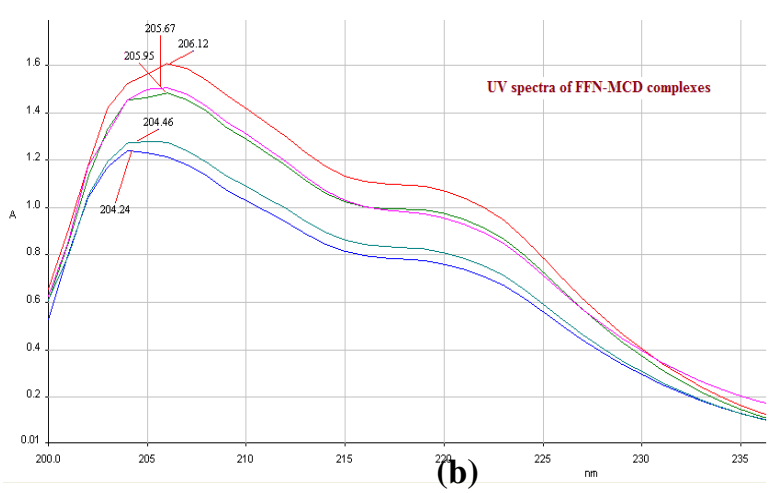

(b)

Figure 11. (a) Phase solubility plot for Fexofenadine Hydrochloride at a different methyl- $\beta$-cyclodextrin concentration in water; (b) UV-VIS spectra Fexofenadine Hydrochloride at a different methyl- $\beta$-cyclodextrin concentration in water

Preparation physical mixtures fexofenadine hydrochloride/cyclodextrin-Samples were prepared by physical mixing of fexofenadine hydrochloride and $\beta$-cyclodextrin and their derivatives. Samples were prepared in a molar ratio of $1: 1$. Their interpretation was performed using FTIR spectroscopy. The spectra of the samples were compared with the spectrum of pure CRS standard of fexofenadine hydrochloride and the spectra of pure uncomplexed cyclodextrins.
Interpretation of fexofenadine hydrochloride by FTIR spectroscopy showed the existence of the three most significant peaks that characterize its structure, namely the peaks at about $3300 \mathrm{~cm}^{-1}$ (hydroxyl groups), $2945 \mathrm{~cm}^{-1}$ (acid $-\mathrm{OH}$ ), and $1680 \mathrm{~cm}^{-1}$ (carbonyl group) ${ }^{12}$. Figure 11 shows the FTIR spectra of pure FFN and its binary physical mixtures, $\beta$-cyclodextrin, and their derivatives in a molar ratio of 1:1. The characteristic peaks of FFN are observed during the comparative analysis of FTIR spectra. The peak at $3300 \mathrm{~cm}^{-1}$ is visible in all complexes but is 
shifted toward higher wavelengths, and it is much wider. $\left(3319 \mathrm{~cm}^{-1}\right.$ FFN- $\beta C D$; $3315 \mathrm{~cm}^{-1}$ FFN-2H $\beta C D$; $3314 \mathrm{~cm}^{-1}$ FFN-m $\left.\beta C D\right)$. Also, a peak at $2945 \mathrm{~cm}^{-1}$ is present and is shifted. $\left(2948 \mathrm{~cm}^{-1}\right.$ FFN- $\beta C D$; $2946 \mathrm{~cm}^{-1} \quad 2 \mathrm{H} \beta C D ; 2943 \mathrm{~cm}^{-1} \quad$ FFN-m $\left.\beta C D\right)$. FTIR spectra show a very low intensity and almost lose the band originating from the carbonyl groups $\left(1680 \mathrm{~cm}^{-1}\right)$. All this indicates that there has been a change in the structure of the molecule that is most likely caused by the formation of intermolecular interactions of cyclodextrin and drugs.

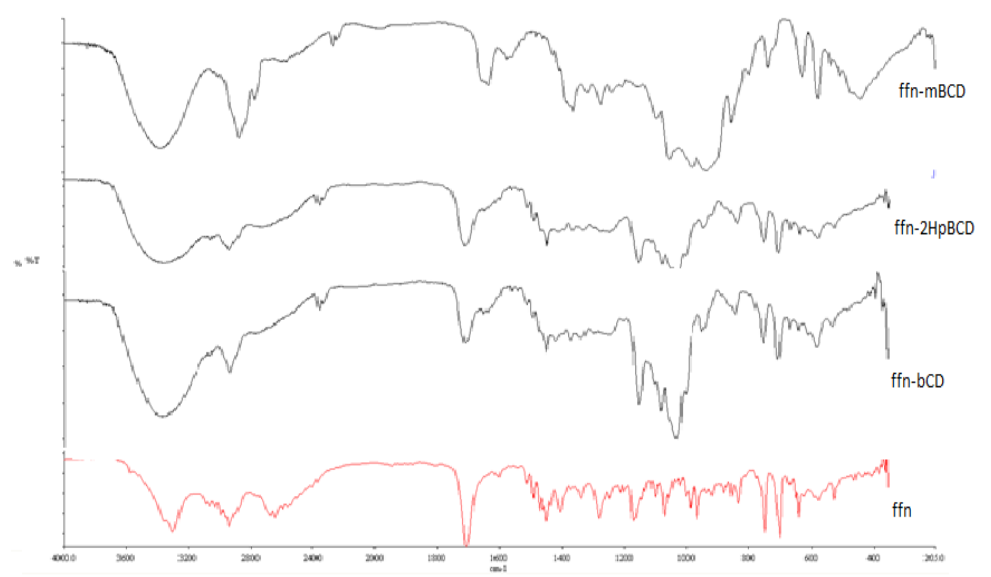

Figure 12. FTIR spectra or a pnysical mixture or rexorenaaıne nyarocnıorıae ana $p$-cycıoaextrin and their derivatives (2-Hydroxypropyl- $\beta$-cyclodextrin and methyl- $\beta$-cyclodextrin) in molar ratio 1:1

\section{Conclusion}

Fexofenadine hydrochloride is a drug that belongs to the group of antihistamines and has a low aqueous solubility of $2.136 \mathrm{mg} / \mathrm{ml}$. The use of cyclodextrin increases the aqueous solubility of fexofenadine hydrochloride and leads to the formation of inclusion complexes with fexofenadine hydrochloride.

$\beta$-cyclodextrin with fexofenadine hydrochloride leads to the formation of A-type phase solubility by Higuchi and Connors. Derivative of $\beta$-cyclodextrin, 2 -hydroxypropyl- $\beta$-cyclodextrin, and methyl- $\beta$ cyclodextrin show $A_{p}$ type of phase solubility with fexofenadine hydrochloride. A physical mixture of fexofenadine hydrochloride and $\beta$-cyclodextrins and their derivatives in a molar ratio of $1: 1$ can lead to the formation of inclusion complexes.

\section{References}

1. M. M. Heravi, V. Zadsirjan, Prescribed drugs containing nitrogen heterocycles: an overview, RSC Adv., 2020, 10, 44247-44311.

2. C. T. Abhiman, J. H. Anantrao, Phase solubility studies of glimepiride with $\beta$-cyclodextrin and hydroxy propyl- $\beta$-cyclodextrin in different $\mathrm{pH}$, Indo American Journal of Pharmaceutical Research, 2017, 7, 604-612.

3. M. M. Al Omari, A. A. Badwan, M. B. Zughul, J. E. Davies, Fexofenadine/Cyclodextrin Inclusion Complexation: Phase Solubility, Thermodynamic, Physicochemical, and Computational Analysis Drug Development and Industrial Pharmacy, 2007, 33, 1205-1215.
4. N. P. Sapkal, V. A. Kilor, B. D. Shewale, K. P. Bhusari, A. S. Daud, Study of the Complexation Behaviour of Fexofenadine with $\beta$-Cyclodextrin, Indian J Pharm Sci., 2010, 72(3), 318-323.

5. S. S. Jambhekar, P. Breen, Cyclodextrins in pharmaceutical formulations I: structure and physicochemical properties, formation of complexes, and types of complex; Drug Discovery Today, 2016, 21, 356-362.

6. H. Dodziuk, Molecules with holes-Cyclodextrins, Cyclodextrins and their complexes, 2006, 1-30.

7. P. Saokham, C. Muankaew, P. Jansook, T. Loftsson, Solubility of Cyclodextrins and Drug/Cyclodextrin Complexes, Journal Molecules, 2018, 23, 1161.

8. M. M. Al Omari, M. B. Zughul, E. D. Davies, A. A. Badwan, Effect of buffer species on the complexation of basic drug terfenadine with $\beta$-cyclodextrin, Journal of Inclusion Phenomena Macrocyclic Chemistry., 2007, 58, 227-235.

9. N. Ojha, B. Prabhakar, Advances in Solubility Enhancement Techniques, Int. J. Pharm. Sci. Rev. Res., 2013, 21, 351-358.

10. M. M. Al Omari, A. A. Badwan, Fexofenadine/Cyclodextrin Inclusion Complexation: PhaseSolubility, Thermodynamic, Physicochemical and Computational Analysis, Drug Development and Industrial Pharmacy, 2007, 33, 1205-1215.

11. S. J. Pandya, T. Y. Pasha, A. Bhandari, J. K. Patel, T. Naitik, T. Upama, Physical Characterization and Enhance the Solubility of Fexofenadine- $\beta$-Cyclodextrin Inclusion Complexes, Current Pharma Research, 2011, 3, 260-266. 